\title{
Penetration characteristics of VLF wave from atmosphere into lower ionosphere*
}

\author{
Shufan Zhao ${ }^{1, \star}$ Xuhui Shen ${ }^{1}$ Weiyan Pan $^{2}$ \\ Xuemin Zhang $^{1}$ and Li Liao $^{3}$ \\ ${ }^{1}$ Institute of Earthquake Science, China Earthquake Administration, Beijing 100036, China \\ ${ }^{2}$ Qingdao Branch, China Research Institute of Radiowave Propagation, Qingdao 266107, China \\ ${ }^{3}$ Institute of Geophysics, China Earthquake Administration, Beijing 100081, China
}

\begin{abstract}
The factors affecting the reflection and transmission coefficient of the ionosphere have been analyzed. These factors include wave frequency, incident angle, geomagnetic inclination, electron density and collision frequency in the ionosphere. The ionosphere refractive index is also analyzed. The ionosphere above $70 \mathrm{~km}$ is considered to be homogeneous and anisotropic, and the reflection and transmission coefficient matrix is calculated using matrix method. Simultaneously the Booker quartic equation is solved to get the refractive index in the ionosphere. The results show that when the wave frequency is higher, it is easier to penetrate into the ionosphere from its bottom boundary and the propagation attenuation in the ionosphere is smaller. TE (traverse electric) wave and TM (traverse magnetic) wave can both penetrate into the ionosphere with a small incident angle, while TE wave can hardly transmit into the ionosphere when the incident angle is large. The transmission coefficient decreases as the geomagnetic inclination increases. TE and TM wave cannot penetrate into the ionosphere at magnetic equator. When the electron collision frequency is higher, it is easier for VLF wave to penetrate into the ionosphere and the attenuation of ordinary wave is weaker, which may be caused by the energy transportation between the waves and the particles. The ordinary $(\mathrm{O})$ wave experiences severer attenuation than extraordinary $(\mathrm{X})$ wave, and $\mathrm{X}$ wave is a penetration mode whereas $\mathrm{O}$ wave is a non-penetration mode in the ionosphere. All the results indicate that VLF wave with higher frequency is easier to penetrate into the ionosphere and to be recorded by the satellites at high latitude. It is hard for ULF and the lower frequency VLF wave to transmit into the ionosphere directly for the severe reflection and attenuation. It may transmit into the ionosphere with a small incident angle due to the nonlinear effect, for example, the interaction between the waves and the particles or cross modulation, and then propagate along the whistle duct with small attenuation. This work may be a preliminary theoretical exploration for the future calculation on the response of ground based VLF artificial transmitter in the ionosphere and further study on the seismic ionosphere coupling model.
\end{abstract}

Key words: VLF wave; low ionosphere; reflection coefficient; transmission coefficient; refractive index CLC number: P315.9 Document code: A

\section{Introduction}

A variety of electromagnetic phenomena associated with earthquakes have been reported in a wide low frequency range, and the satellites have recorded prominent signals of frequency ranging from ULF to ELF/VLF in the ionosphere and the lower magnetosphere (Larkina et al, 1983; Chmyrec et al, 1989; Parrot and Mogilevsky, 1989; Serebryakova et al, 1992; Molchanov et al, 1993;

\footnotetext{
* Received 9 September 2009; accepted in revised form 18 December 2009; published 10 June 2010.

4 Corresponding author. e-mail: zsf2008bj@126.com

(C) The Seismological Society of China and Springer-Verlag Berlin Heidelberg 2010
}

Parrot, 1994; Pulinets and Legen'ka, 2003). To support current missions such as DEMETER that have been deployed specifically in search of seismogenic electromagnetic signals, the group designed theoretical work to understand the propagation of such signals from the Earth surface to the topside ionosphere. Theoretical calculations have been proposed to analyze the seismic electromagnetic wave intensities (Molchanov et al, 1995; Grimal'skii and Rapoport, 2000). However, all these works have not clearly shown the effects of the geomagnetic field on the wave propagation. Bortnik and Bleier (2004) considered the effect of geomagnetic field, but they only analyzed the seismic electromagnetic 
wave intensities at the ELF and ULF band. It is well-known that low frequency electromagnetic signals experience smaller decay underground than high frequency, however, in the ionosphere, only relative higher frequency can penetrate, so it is difficult for ultra low frequency signals to be recorded directly by satellites. The VLF signals make a bridge for us to understand the direct propagating process of low frequency electromagnetic signals from the Earth to the ionosphere. Furthermore, there are many VLF transmitters navigating for submarine, so when the satellites are flying over around the transmitters, they can receive the signals from these transmitters in local nighttime (Molchanov et al, 2006). That is to say we can take advantage of these manual VLF signals to modify and testify our model of electromagnetic wave propagation at very low frequency. In this paper, attention was focused on VLF band. The reflection and transmission coefficient were calculated using matrix method, simultaneously refractive index was calculated by solving a complex coefficient quartic equation for homogeneous sharp boundary ionosphere, which is of great significance for our further calculation on the response of ground-based VLF artificial transmitter in the ionosphere and future study on the seismic ionosphere coupling model.

\section{Theoretical derivation}

The coordinate system $(\theta, \varphi, r)$ is shown in Figure 1. A plane wave is incident on anisotropic ionosphere which is assumed to vary only in the $r$ direction. The incident plane is the $\theta-r$ plane. The direction of the Earth's magnetic field is $(l, m, n)$.

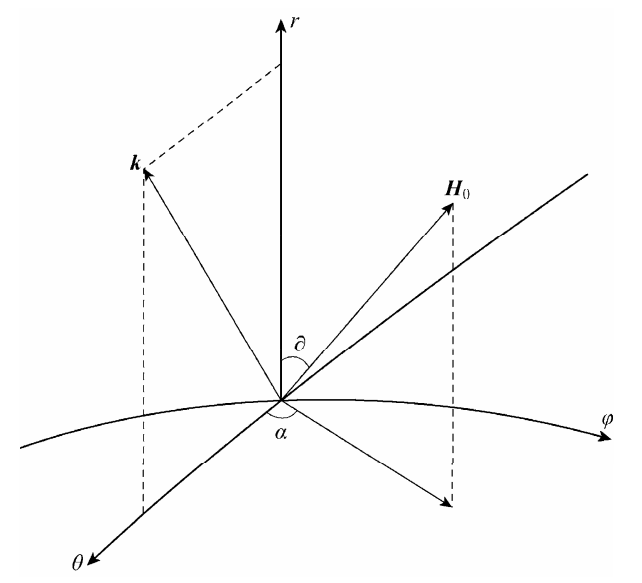

Figure 1 Geometry of the problem. The plane of incidence is the $\theta-r$ plane. The direction of cosines of the Earth's magnetic field $\boldsymbol{H}_{0}$ is $(l, m, n)$.
Since the solution space contains gradients that vary quickly over the spatial scale of a wavelength, we resort to a full-wave solution. With field variables which vary with time as $\mathrm{e}^{-\mathrm{j} \omega t}$, electromagnetic wave fields in the ionosphere satisfy the following Maxwell's equations (Budden, 1961):

$$
\nabla \times \boldsymbol{H}=\mathrm{j} \omega \varepsilon_{0}(\boldsymbol{I}+\boldsymbol{M}) \cdot \boldsymbol{E}
$$

and

$$
\nabla \times \boldsymbol{E}=-\mathrm{j} \omega \mu_{0} \boldsymbol{H},
$$

where $\varepsilon_{0}, \mu_{0}$ and $\boldsymbol{I}$ denote the permittivity and permeability of free space, and the unit matrix. The effect of ions on the wave propagation is ignored and only electrons effect is considered which is reasonable at VLF frequency band because wave frequency is much greater than ion gyro-frequency but equivalent to the electron gyro-frequency. The magnetic susceptibility matrix $\boldsymbol{M}$ (Yeh and Liu, 1972) is given by

$$
\begin{gathered}
\boldsymbol{M}=-\frac{X}{U\left(U^{2}-y^{2}\right)} \cdot \\
{\left[\begin{array}{ccc}
U^{2}-l^{2} y^{2} & \mathrm{i} n y U-l m y^{2} & -\mathrm{i} m y U-\ln y^{2} \\
-\mathrm{i} n y U-l m y^{2} & U^{2}-m^{2} y^{2} & \mathrm{i} l y U-m n y^{2} \\
\mathrm{i} m y U-\ln y_{\alpha}^{2} & -\mathrm{i} l y U-m n y_{\alpha}^{2} & U^{2}-n^{2} y^{2}
\end{array}\right]}
\end{gathered}
$$

with $U=1+\mathrm{j} Z, Z=v / \omega, X=\left(\omega_{p} / \omega\right)^{2}, \omega_{p}^{2}=e^{2} N / m \varepsilon_{0}, \omega_{h}=e B / m$, $Y=\left|\omega_{h} / \omega\right|$. Where $\omega_{p}$ is electron plasma angular frequency, $\omega_{h}$ is electron gyro angular frequency, $e$ is electron charge $(\mathrm{C}), m$ is electron mass $(\mathrm{kg}), N$ is electron number density $\left(\mathrm{m}^{-3}\right), v$ is electron collision frequency.

If the medium is divided into a number of thin homogeneous slabs (Figure 2), there are two up-going waves and two down-going waves in each slab.

Maxwell equation is solved in the multilayered anisotropical media using the propagation matrix method (Chew, 1990), and the generalized reflection and transmission coefficient matrixes $\tilde{\boldsymbol{R}}$ and $\tilde{\boldsymbol{S}}$ are calculated.

$$
\left[\begin{array}{c}
\tilde{\boldsymbol{S}} \\
\mathbf{0}
\end{array}\right]=\boldsymbol{a}_{N}^{-1} \cdot\left(\prod_{j=n}^{1} \boldsymbol{P}_{j}\left(r_{j}-r_{j-1}\right)\right) \cdot \boldsymbol{a}_{0} \cdot\left[\begin{array}{c}
\boldsymbol{I} \\
\tilde{\boldsymbol{R}}
\end{array}\right]
$$

where $\boldsymbol{a}_{j}=\left[\boldsymbol{a}_{1 j}, \boldsymbol{a}_{2 j}, \boldsymbol{a}_{3 j}, \boldsymbol{a}_{4 j}\right](j=0, N)$ is a $4 \times 4$ matrix consisting of four eigenvectors of $\boldsymbol{T}_{j} ; \boldsymbol{T}_{j}$ is the state matrix of the $j$ th layer, whose elements are determined by electron density, collision frequency, geomagnetic field, incident angle and so on; $\boldsymbol{a}_{N}^{-1}$ is the inverse of $\boldsymbol{a}_{N}$; $\boldsymbol{P}_{j}\left(r_{j}-r_{j-1}\right)$ is the propagation matrix of the $j$ th layer, which is determined by the eigenvectors matrix and the 


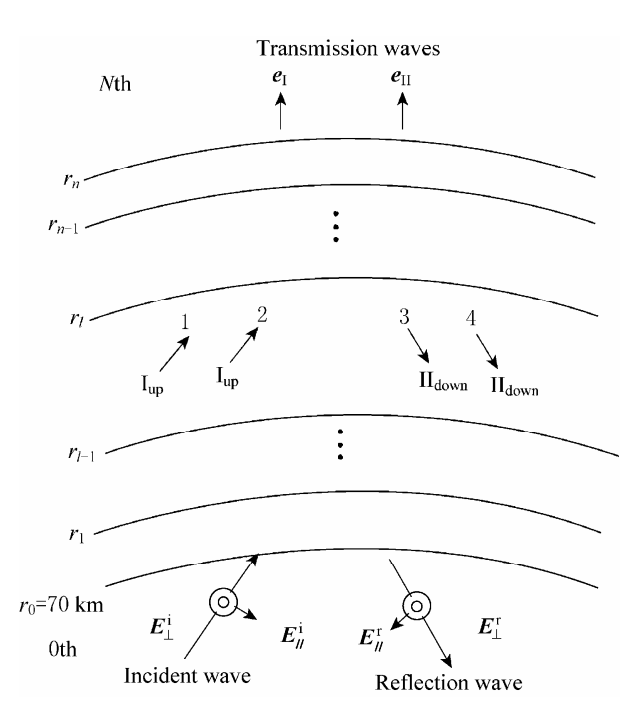

Figure 2 Reflection and transmission waves in the anisotropical spherical multilayered ionosphere.

height of the layer. For

$$
\tilde{\boldsymbol{R}}=\left[\begin{array}{ll}
R_{\mathrm{I}, \mathrm{I}} & R_{\mathrm{I}, \mathrm{II}} \\
R_{\mathrm{II}, \mathrm{I}} & R_{\mathrm{II}, \mathrm{II}}
\end{array}\right]
$$

and

$$
\tilde{\boldsymbol{S}}=\left[\begin{array}{ll}
S_{\mathrm{I}, \mathrm{I}} & S_{\mathrm{I}, \mathrm{II}} \\
S_{\mathrm{II}, \mathrm{I}} & S_{\mathrm{II}, \mathrm{II}}
\end{array}\right],
$$

the four elements respectively represent the reflection and transmission between the I wave and the II wave, the characteristic waves in the media. As we have known, the characteristic waves in the isotropical atmosphere are TM (transverse magnetic) wave and TE (transverse electronic) wave; the characteristic waves in the anisotropical ionosphere are $\mathrm{O}$ (ordinary) wave and $\mathrm{X}$ (extraordinary) wave. For example, $R_{\mathrm{I}, \mathrm{II}}$ stands for the reflection coefficient that TM wave is reflected into TE wave, $S_{\mathrm{I}, \mathrm{II}}$ means the transmission coefficient that TM wave transmits into $\mathrm{X}$ wave.

The eigenvalues and eigenvectors of matrix $\boldsymbol{T}$ can be easily calculated when electron density, collision frequency, geomagnetic field, incident angle are given, then after that $\tilde{\boldsymbol{R}}$ and $\tilde{\boldsymbol{S}}$ can be calculated using equation (4).

But a serious problem is arisen that when the amount of the layer is up to 5 , instability problems caused by the iterative multiplication of propagation matrix of each layer are encountered. How to overcome the numerical swamping will be discussed in my ensu- ing papers. In this paper reflection and transmission coefficients in the homogeneous half-space are solved by matrix method.

When $r_{n}=r_{0}$, the total propagation matrix $\boldsymbol{P}$ degenerates into a unit matrix. $\boldsymbol{a}_{0}$ and $\boldsymbol{a}_{N}$ are the eigenvector matrixes of the atmosphere and ionosphere separately. Solving the matrix equation (4) with four equations and four unknowns, then every element of the reflection and transmission coefficient matrixes can be obtained.

Simultaneously we solve the Booker quartic equation (Booker, 1939) to get the refractive index $D$ in the ionosphere.

$$
b_{4} D^{4}+b_{3} D^{3}+b_{2} D^{2}+b_{1} D+b_{0}=0
$$

where

$$
\begin{gathered}
b_{4}=U\left(U^{2}-Y^{2}\right)+X\left(n^{2} Y^{2}-U^{2}\right), \\
b_{3}=2 \ln S X Y^{2} \\
b_{2}=-2 U(U-X)\left(C^{2} U-X\right)^{2}+2 Y^{2}\left(C^{2} U-X\right)+ \\
X Y^{2}\left(1-C^{2} n^{2}+S^{2} l^{2}\right), \\
b_{1}=-2 C^{2} \ln S X Y^{2}
\end{gathered}
$$

and

$$
b_{0}=(U-X)\left(C^{2} U-X\right)^{2}-C^{2} Y^{2}\left(C^{2} U-X\right)-l^{2} S^{2} C^{2} X Y^{2} .
$$

If the refractive index has great real part, it means that the ionosphere has high dispersion to this characteristic wave. The great imaginary part means that this characteristic wave attenuates rapidly in the ionosphere and is a 'non-propagating mode'.

\section{Simulation results}

If no special explanation is given, the geomagnetic intensity is $5 \times 10^{-5} \mathrm{~T}$ and the electron density is $10^{10} / \mathrm{m}^{3}$. When the dependence on wave frequency is discussed, geomagnetic inclination and the electron collision frequency are separately set to be $45^{\circ}$ and $10^{6} / \mathrm{s}$. When the dependence on geomagnetic inclination is discussed, the wave frequency is fixed to the value $19.8 \mathrm{kHz}$; and the wave is vertically incident.

The calculation results of the reflection coefficient are shown in Figures 3 and 4. Figure 3 shows the modulus of the principal diagonal elements of the reflection coefficient matrix varying in wave frequency with different incident angles.

Comparing these four figures ( $3 a, 3 b, 3 c$ and $3 d$ ), we can see that $R_{11}, R_{22}$ both reduce as the wave frequency increases. $R_{22}$ increases while $R_{11}$ decreases as 
the increase of incident angle, and they are equal when the incident angle is zero. When the incident angle is great, $R_{22}$ nearly reach to 1 . That is to say, TE wave cannot penetrate into the ionosphere for severer reflection, while TM wave is easier to penetrate into the ionosphere when the incident angle is large.

The values of non-principal diagonal elements $R_{12}$ and $R_{21}$ increase with frequency as shown in Figure 4. $R_{12}$ and $R_{21}$ have no significant change with the incident
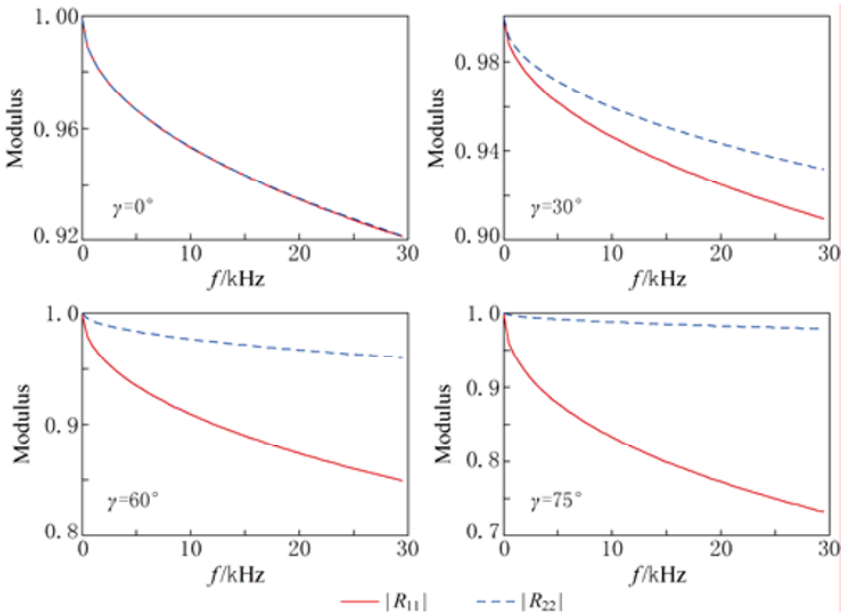

Figure 3 Dependence on frequency $(f)$ of the modulus of principal diagonal elements $\left(R_{11}\right.$ and $\left.R_{22}\right)$ of reflection matrix with incident angle $\gamma$ being $0^{\circ}, 30^{\circ}, 60^{\circ}$ and $75^{\circ} . R_{11}$ stands for reflection coefficient of TM wave being reflected into TM wave, and $R_{22}$ denotes reflection coefficient of TE wave being reflected into TE wave.
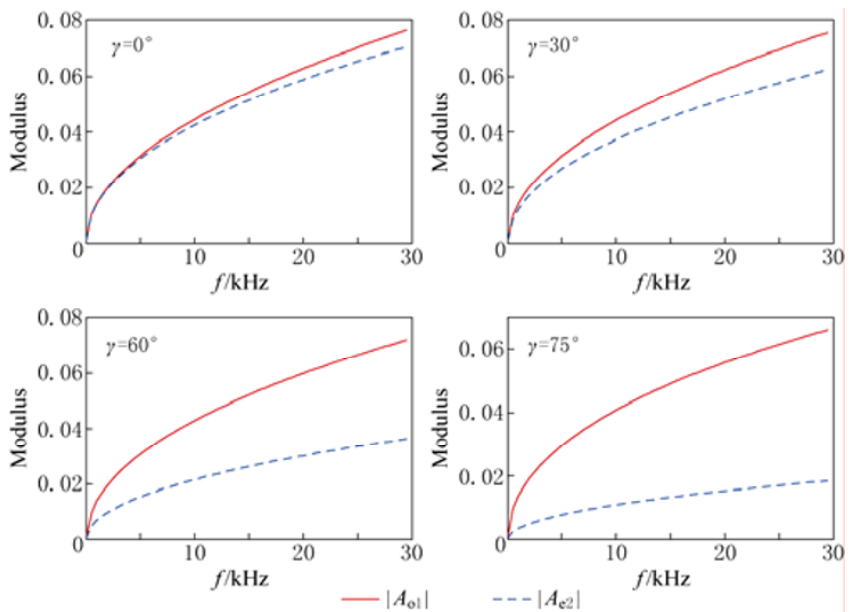

Figure 5 Dependence on frequency $(f)$ of the modulus of principal diagonal elements $\left(A_{\mathrm{o} 1}\right.$ and $\left.A_{\mathrm{e} 2}\right)$ of transmission matrix with incident angle $\gamma$ being $0^{\circ}, 30^{\circ}, 60^{\circ}$ and $75^{\circ} . A_{\mathrm{o} 1}$ and $A_{\mathrm{e} 2}$ stand for the transmission coefficient of TM wave converted into ordinary wave and the transmission coefficient of TE wave converted into extraordinary wave, respectively. angle. The results of the transmission coefficient are shown in Figures 5 and 6.

Figures 5 and 6 show the transmission coefficients increasing with frequency. When the incident angle increases, $A_{\mathrm{o} 1}$ and $A_{\mathrm{e} 1}$ decrease slightly, but $A_{\mathrm{e} 2}$ and $A_{\mathrm{o} 2}$ decrease significantly. To sum up, TE wave can penetrate only when the incident angle is small, in accordance with the results of reflection coefficient above.
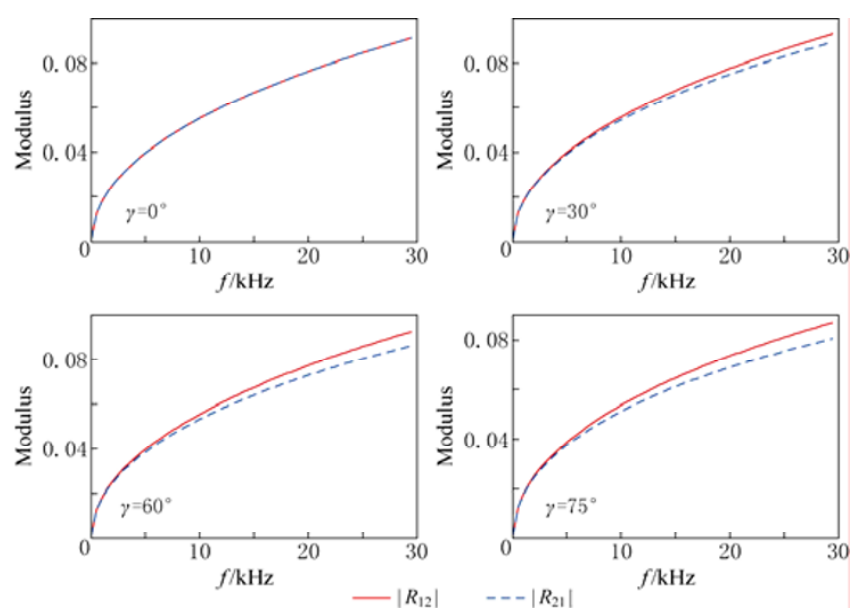

Figure 4 Dependence on frequency $(f)$ of the modulus of non-principal diagonal elements $\left(R_{12}\right.$ and $\left.R_{21}\right)$ of reflection matrix with incident angle $\gamma$ being $0^{\circ}, 30^{\circ}, 60^{\circ}$ and $75^{\circ} . R_{12}$ stands for reflection coefficient of TM wave being reflected into TE wave, and $R_{21}$ denotes reflection coefficient of TE wave being reflected into TM wave.
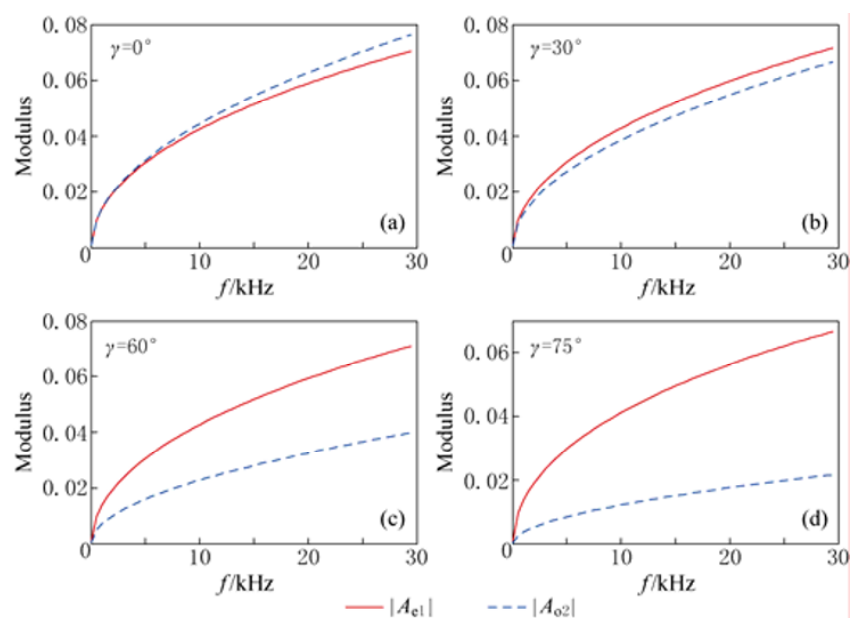

Figure 6 Dependence on frequency $(f)$ of the modulus of non-principal diagonal elements $\left(A_{\mathrm{e} 1}\right.$ and $\left.A_{\mathrm{o} 2}\right)$ of transmission matrix with incident angle $\gamma$ being $0^{\circ}, 30^{\circ}, 60^{\circ}$ and $75^{\circ} . A_{\mathrm{el}}$ stands for the transmission coefficient of TM wave converted into extraordinary wave, and $A_{\mathrm{o} 2}$ denotes the transmission coefficient of TE wave converted into ordinary wave. 
Then the dependence of reflection and transmission coefficients on geomagnetic inclination with different collision frequency is discussed when the wave is vertically incident, as shown in Figure 7.
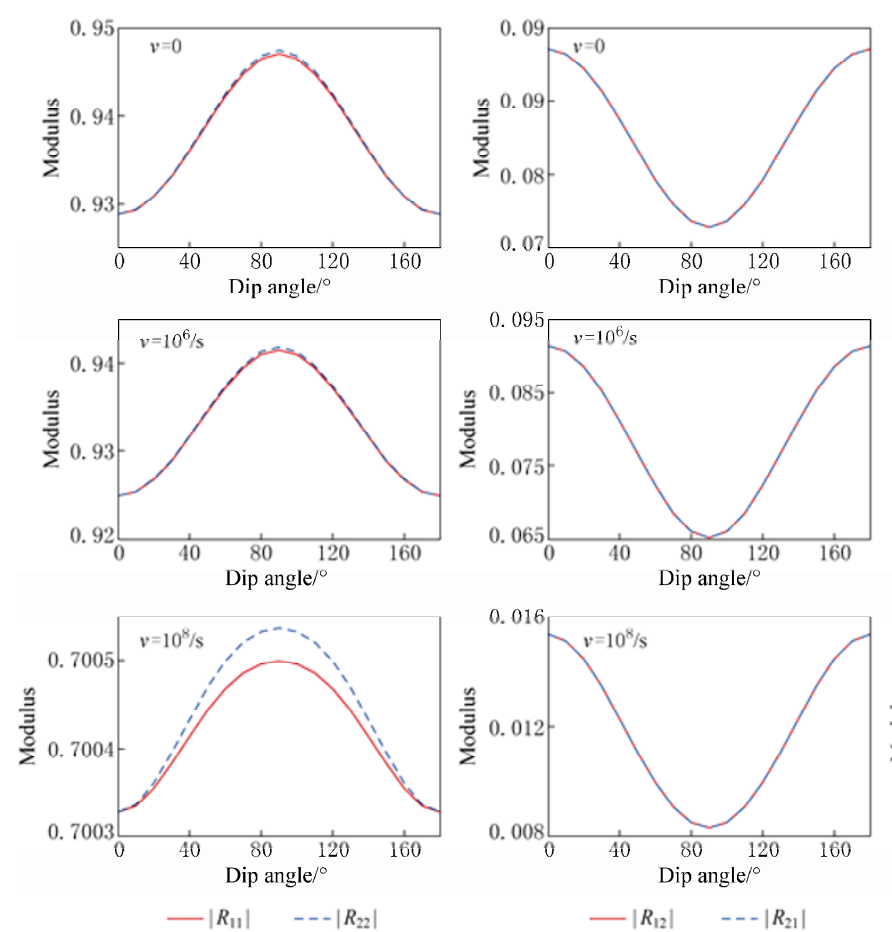

The reflection coefficients all decrease, while transmission coefficients all increase dramatically when the electron collision frequency changes from $10^{6} / \mathrm{s}$ to $10^{8} / \mathrm{s}$. $R_{11}$ and $R_{22}$ increase while $R_{12}, R_{21}$ decrease, and $A_{\mathrm{o} 1}, A_{\mathrm{e} 2}$,
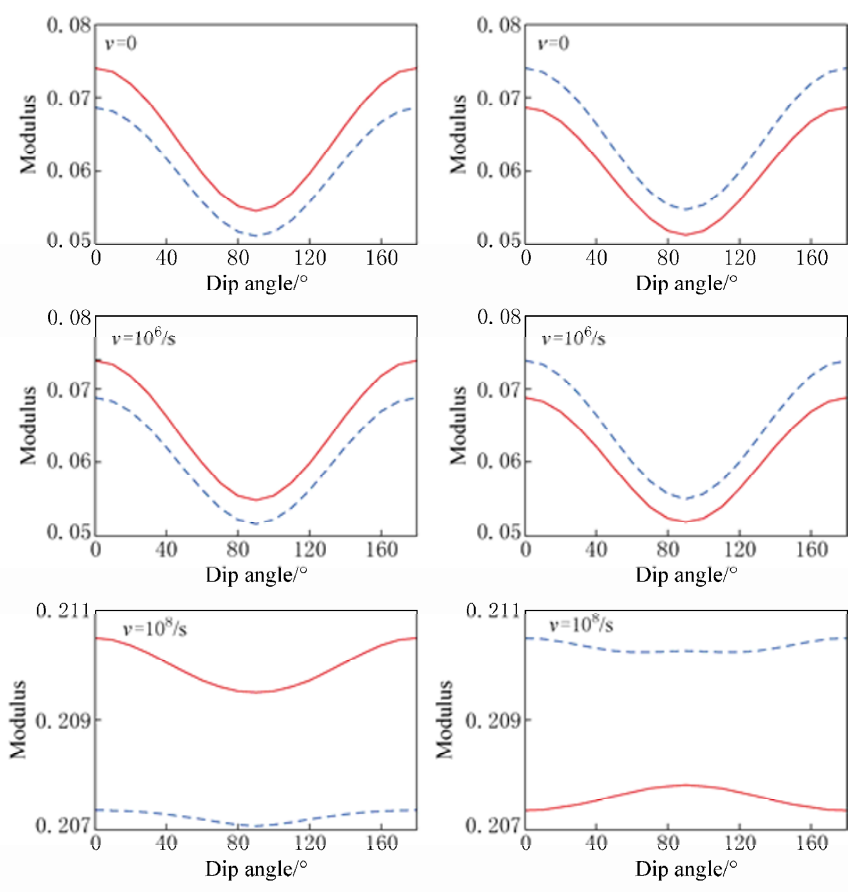

$-\left|A_{\mathrm{o} 1}\right| \quad---\left|A_{\mathrm{e} 2}\right|$

$-\left|A_{\mathrm{e} 1}\right| \quad---\left|A_{02}\right|$

Figure 7 Dependence on dip angle of reflection and transmission coefficient with collision frequency $v$ being $0,10^{6} / \mathrm{s}$ and $10^{8} / \mathrm{s}$.
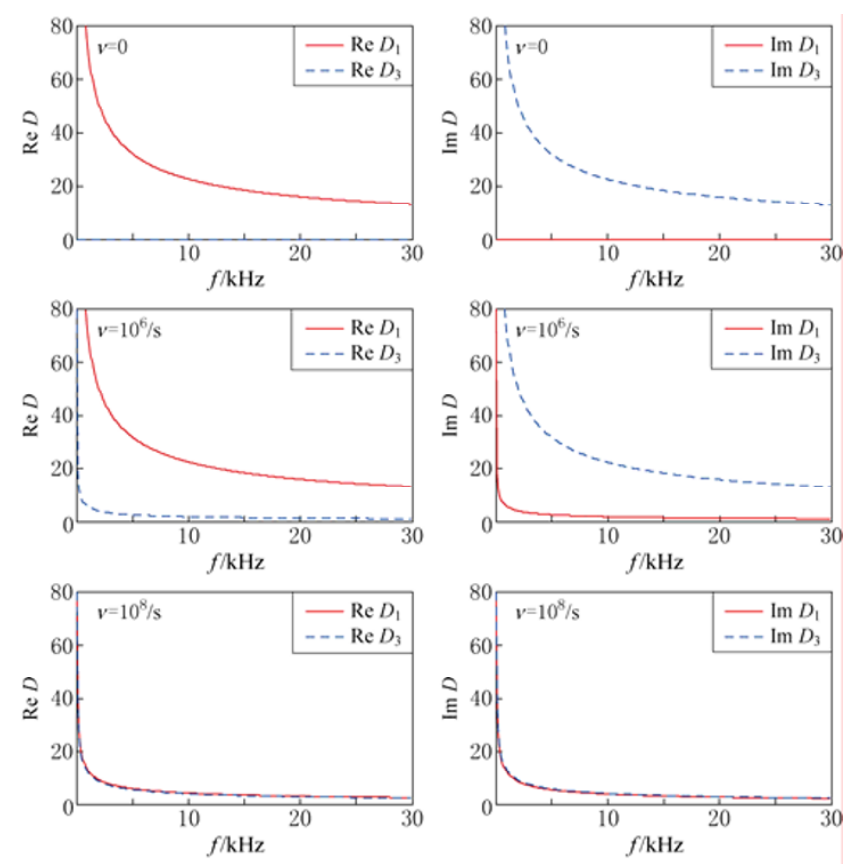

Figure 8 Real and imaginary part of the refractive indices of extraordinary wave $\left(D_{1}\right)$ and ordinary wave $\left(D_{3}\right)$ versus frequency, with collision frequency $v$ being $0,10^{6} / \mathrm{s}$ and $10^{8} / \mathrm{s}$.
$A_{\mathrm{e} 1}$ and $A_{\mathrm{0} 2}$ all decrease as the dip angle increasing from $0^{\circ}$ to $90^{\circ}$, when electron collision frequency $(v)$ is not very great, but they have no significant change trend when electron collision frequency is great. The variation trends from $90^{\circ}$ to $180^{\circ}$ are exactly symmetrical. It needs to be pointed out that dip angle here is defined as the complementary angle of the geomagnetic inclination. That is to say, when the geomagnetic inclination is $0^{\circ}$, at the magnetic equator, the most significant reflection and weakest transmission happens and the waves can hardly penetrate into the ionosphere.

We now discuss the propagation characteristic of the $\mathrm{O}$ wave and $\mathrm{X}$ wave in the ionosphere. As shown in Figure 8 , the real parts and imaginary parts of the refractive indices of $\mathrm{O}$ wave and $\mathrm{X}$ wave at different collision frequency are calculated, respectively, with the change of wave frequency. $D_{1}$ is described as the refractive index of $\mathrm{X}$ wave, and $D_{3}$ as $\mathrm{O}$ wave.

From the results we can find that at VLF band the $\mathrm{X}$ wave has the refractive index with great real part and small imaginary, opposite to $\mathrm{O}$ wave. The real parts and imaginary parts of the refractive indices of the two 
waves decrease as the increase of wave frequency. The imaginary part of $\mathrm{X}$ wave increases with the collision frequency and equals to zero when the collision frequency is 0 , which means it is a 'propagating mode' with little attenuation. The imaginary part of $\mathrm{O}$ wave decreases as the increase of collision frequency and equals to $\mathrm{X}$ wave when collision frequency is great enough.

\section{Discussion and conclusions}

We have performed plenty of analysis on the factors influencing the reflection and transmission coefficient of the ionosphere, such as wave frequency, incident angle, geomagnetic inclination and electron collision frequency in the ionosphere. The refractive index is also analyzed in this paper. We calculated the reflection and transmission coefficient matrix using matrix method (Chew, 1990). Simultaneously we solved the Booker quartic equation (Booker, 1939) to get the refractive index in the ionosphere. This work may be the preliminary theoretical exploration for the further calculation on the response of ground-based VLF artificial transmitter in the ionosphere and future study on the seismic ionosphere coupling model. We reach some conclusions.

1) The reflection coefficients of TM wave reflected into TM wave $\left(R_{11}\right)$ and TE wave reflected into TE wave $\left(R_{22}\right)$ decrease as the wave frequency increases. That is to say, it is easier for waves of higher frequency to penetrate into the ionosphere. $R_{11}$ equals to $R_{22}$ when incident angle is $0^{\circ} . R_{11}$ decreases, on the contrary, $R_{22}$ increases as the incident angle increases. It can be found that TE wave can hardly penetrate into the ionosphere when the incident angle is large. $R_{11}$ and $R_{22}$ decrease as the geomagnetic inclination increases from $0^{\circ}$ to $90^{\circ}$, and the variation trends from $180^{\circ}$ to $90^{\circ}$ are exactly symmetrical. When the geomagnetic inclination is $0^{\circ}$, at magnetic equator, the most significant reflection appears and the waves can hardly penetrate into the ionosphere. $R_{11}$ and $R_{22}$ decrease as the electron collision frequency increases. This may be caused by the energy transport between the waves and the particles.

2) The value of reflection coefficients of TM wave reflected into TE wave $\left(R_{12}\right)$ and TE wave reflected into TM wave $\left(R_{12}\right)$ are very small. They increase slightly as the wave frequency and the geomagnetic inclination increase, and have no significant change with the incident angle. They decrease slightly as the increase of the collision frequency. It can be concluded that TE wave and TM wave have unconspicuous coupling at VLF band.

3) The transmission coefficients of TM wave transmitted into ordinary wave $\left(A_{\mathrm{o} 1}\right)$ and TE wave converted into extraordinary wave $\left(A_{\mathrm{e} 2}\right)$ as well as the transmission coefficients of TM wave transmitted into extraordinary wave $\left(A_{\mathrm{e} 1}\right)$ and TE wave converted into ordinary wave $\left(A_{02}\right)$ all increase as wave frequency and the geomagnetic inclination increase, which is in accord with the analysis of reflection coefficients above. $A_{01}$ and $A_{\mathrm{e} 1}$ have no significant change with the incident angle, while $A_{\mathrm{e} 2}$ and $A_{\mathrm{0} 2}$ decrease as the increase of the incident angle. So it is easier for TE and TM waves to transmit into the ionosphere at high latitude with high wave frequency in a small incident angle. $A_{\mathrm{o} 1}, A_{\mathrm{e} 2}, A_{\mathrm{e} 1}$ and $A_{02}$ increase sharply when the electron collision frequency changes from $10^{6} / \mathrm{s}$ to $10^{8} / \mathrm{s}$, which may be caused by the interaction between the waves and the particles.

4) In VLF band, $X$ wave has large real part in its refractive index, indicating that $\mathrm{X}$ wave experiences fierce dispersion. The real part decreases sharply when the electron collision frequency changes from $10^{6} / \mathrm{s}$ to $10^{8} / \mathrm{s}$. The imaginary part of $\mathrm{X}$ wave refractive index is small, which means $X$ wave experiences small attenuation, and it increases as the collision frequency. So $\mathrm{X}$ wave is also called as whistler mode, which is a 'propagating mode'. $\mathrm{O}$ wave has the contrary characteristics, which has severe attenuation as a 'non-propagating mode'. The real parts and the imaginary parts of the refraction indices of the two waves decrease dramatically as the increase of wave frequency, which is in accordance with the fact that ionosphere has less influence on the wave propagation when the frequency is higher.

Acknowledgements This work was supported by Chinese National Science and Technology Support Program (2008BAC35B01) and Basic Research Project from Institute of Earthquake Science, China Earthquake Administration (02092408).

\section{References}

Booker H G (1939). The propagation of wave packets incident obliquely on a stratified doubly refracting ionosphere. Phil Trans A 237: 411-451.

Budden K G (1961). Radio Waves in the Ionosphere. Cambridge University Press, London and New York, 389-390.

Chew W C (1990). Waves and Field in Inhomogeneous Media. Van Nostrand Reinhold, New York, 109-114.

Chmyrec V M, Isaev N V, Bilichenko S V and Stanev G (1989). Observation by space-borne detectors of electric fields and hydromagnetic waves in the ionosphere over an earthquake center. Phys Earth Planet Inter 57: $110-114$. 
Bortnik J and Bleier T (2004). Full wave calculation of the source characteristics of seismogenic electromagnetic signals as observed at LEO satellite altitudes. Eos Trans AGU 85(47), Fall Meet. Suppl., Abstract T51B-0453.

Grimal'skii V V and Rapoport Yu G (2000). Penetration of electrostatic field from surface sources into the lower ionosphere. Kinematics and Physics of Celestial Bodies 16: 5-10.

Larkina V I, Nalivayko A V, Gershenzon N I, Gokhberg M B, Liperovskii V A and Shalimov S L (1983). Intercosmos-19 observation of VLF emissions associated with seismic activity. Geomagn Aeron 23: 842-846 .

Molchanov O A, Hayakawa M and Rafalsky V A (1995). Penetration characteristics of electromagnetic emissions from an underground seismic source into the atmosphere, ionosphere, and magnetosphere. J Geophys Res 100: 1691-1 712 .

Molchanov O A, Mazhaeva O A, Golyavin A N and Hayakawa M (1993). Observation by Intercosmos-24 satellite of ELF-VLF electromagnetic emissions associated with earthquakes. Ann Geophys 11: 431-440.

Molchanov O A, Rozhnoi A, Solovieva M, Akentieva O, Berthelier J J, Parrot M, Lefeuvre F, Biagi P F, Castellana L and Hayakawa M (2006). Global diagnostics of the ionospheric perturbations related to the seismic activity using the VLF radio signals collected on the DEMETER satellite. Nat Hazards Earth Syst Sci 6: 745-753.

Parrot M (1994). Statistical study of ELF/VLF emissions recorded by a low altitude satellite during seismic events. J Geophys Res 99: 23 339-23 347.

Parrot M and Mogilevsky M M (1989). VLF emissions associated with earthquakes and observed in the ionosphere and the magnetosphere. Phys Earth Planet Inter 57: 86-99.

Pulinets S A and Legen'ka A D (2003). Spatial-temporal characteristics of the large scale disturbances of the electron concentration observed in the F-region of the ionosphere before strong earthquake. Cosm Res 41: 221-229.

Serebryakova O N, Bilichenko S V, Chmyrev V M, Parrot M, Rauch J L, Lefeuvre F and Pokhotelov O A (1992). Electromagnetic ELF radiation from earthquake regions as observed by low-altitude satellites. Geophys Res Lett 19: 91-94.

Yeh K C and Liu C H (1972). Theory of Ionospheric Waves. Academic Press, New York, 136-139. 